\title{
Index to Volume 6
}

(Numbers followed by asterisk refer to Society Abstracts)

Aagenæs, $\varnothing .59^{*}, 65^{*}$

ABH substances 853

Aceto, T., Jr. 111

Acetylcholine 231, 693

Acetylcholinesterase, mitochondrial 38

Acid-base homeostasis 789

Acid hydrolase 752

Acidosis 43, 584, 789

Adipose tissue 73, 211

Adlard, B. P. F. 38

Adolescence 32,643

Age 32

Åkerblom, H. K. 54*

Albumin 136, 246

Alexiou, D. $51^{*}$

Amino acid(s) 659, 713, 789

Amino acid metabolism 622, 797

p-Aminohippurate 600

Amirhakimi, G. H. 743

Ammonia 797

Amniocentesis $504,720,853$

Amniotic fluid 659

$\alpha$-Amylase 880

Andersen, E. 52*

Andersen, H. $62^{*}$

Andersen, V. 52*

Ando, T. 576

Andreotti, G. $65^{*}, 880$

Androstenedione 111

Antibody 779

Antonowicz, I. 803

Aoyama, T. 622

Aperia, A. 56*, 64*

Appiah, K. E. 43

Arcilla, M. B. 853

Arginase 548

Aspartate transcarbamylase 682

ATPase 616

Atropine 693

Auld, P. A. M. 158

Auricchio, S. 832

Bachmann, C. 56 *

Baerlocher, K. 54*

Bakay, B. 705

Bakken, A. F. 197

Baratz, R. A. 584

Barbanti-Brodano, G. 52*

Barbarani, V. 60*

Barnes, N. 576
Barr, D. G. D. 521

Barratt, T. M. 57*

Bashan, N. 55*

Battaglia, F. C. 182

Baum, J. D. 868

Baumgartner, R. 56*

Beatty, C. H. 813

Berg, U. 56*

Bergadá, C. 634

Berglund, G. 53*

Berra, B. $63^{*}, 66^{*}$

Berthoux, F. 57*

Bicarbonate 495

Bicarbonate reabsorption 859

Bierich, J. R. 54*

Bilirubin 136, 197, 246

Biochemical development 773

Bläker, F. W. 51*

Bleyl, U. 57*

Blum, D. 57*

Bocek, R. M. 813

Boda, D. 57*

Body water 495

Bone 743

Boon, D. A. 111

Borrone, C. $63^{*}, 66^{*}$

Bossi, E. 765

Bottini, E. 53*

Bowe, E. T. 584

Bradley, K. H. 649

Brady, R. N. 119

Brady, R. O. 720

Bradykinin 231, 693

Brain 38, 119, 182, 675, 720, 758

Brain injury 203

Bratteby, L.-E. 58*

Brechbühler, T. 56*

Brent, R. L. 822

Brenton, D. P. 55*

Brill, A. $61^{*}$

Broberger, O. 56*, 64*

Brockmann, U. 487

Brügmann, G. 54*

Buggiani, O. 63*

Burnard, E. D. 61*

Bryson, M. F. 743

Calcium 43, 743

Carbamyl phosphate synthetase 682

Carbon dioxide 584
Cardiac output 158

Catch-up growth 521

Catecholamine 887

Cattaneo, F. 60*

Caul, J. 136

Cavallo, L. 58*

Celiac disease 521

Cell biology 563

Cerebellum 682

Cerebral oxygen consumption 182

Chan, J. C. M. 789

Chantler, C. 57*

Chase, G. 239

Chase, T. N. 730

Chattas, A. 54*

Chiang Teng, C. 659

Children 239

Chloramphenicol 687

Cholestasis 100

Cholesterol 736

Christel, D. 60*

Christiansen, P. A. 713

Chromosomal aberrations 514

Ciccimarra, F. 832

Citrulline 252

Citrullinemia 626

Cividalli, G. 51*

Clark, J. 239

Clomiphene citrate 162

Cole, C. H. 616

Colwill, J. R. 182

Conen, P. E. 514

Congenital enzyme deficiencies 832

Congenital heart disease 151

Connor, J. D. 576

Conrad, G. W. 563

Conrad, M. E. 900

Cook, J. G. H. 66*

Cord blood 593

Cordero, L. 81

Cottofava, F. 62*

Cottom, D. 576

Crawhall, J. C. 504

Cukor, P. 246

Cyclic AMP 552

Cystathionase 538, 773

Cystathionine 172, 538, 773

Cystathionine synthase 187, 538

Cyst(e)ine 172, 538, 649

Cystic fibrosis of the pancreas 26, 616, 803 
Cystinosis 649

Cytochrome C 100

Cytochrome oxidase 687

Dagna-Bicarelli, F. 62*

Danesh, B. J. N. Z. $61^{*}$

Daniel, S. S. 584

Davis, C. E. 643

Davis, S. D. 779

Dechavanne, M. 57*

de Chwojnik, M. F. 634

Dehydration 219

Dehydroepiandrosterone 111

Dell, R. B. 495

Del Principe, D. 58*

Denning, C. R. 26

Desnick, R. 56*

Developmental biochemistry 119, 197, 538, 682

Developmental neurology 887

Developmental physiology $38,43,81,182$, $231,600,634,758,859$

DiGeorge, A. M. 843

Digestion 832, 880

Digilio, G. 58*

1,25-Dihydroxycholecalciferol 552

Dilinoleyl lecithin 701

Dirks, J. H. 616

Dobbing, J. 38

Don, N. A. $66^{*}$

Donnell, G. 576

Dopamine 151, 887

Dorfman, A. 563

Down's syndrome 10,730

Doxiadis, S. 5 I *

Drack, E. 64*

Dubois, J. 57*, 219

Ductus arteriosus 231, 693

Durand, P. 63*

Durant, J. L. 187

Dussault, J. H. 894

Dwyer, D. 813

Dyer, N. $61^{*}$

Edelmann, C. M., Jr. 859

Eidelman, A. I. 81

Ekert, H. 57*

Electroencephalograph 203

Electrolyte 219

Ellis, W. G. 720

Enriquez, A. R. 664

Ente, G. 246

Epinephrine 151

Epiphyseal growth 521

Epstein, C. J. 720

Erenberg, A. 894

Erythroblastosis fetalis 487

Erythrocyte deformation 593

Erythrocyte(s) 10, 487, 548, 616, 705, 736, 900

Erythromycin 687
Extracellular fluid 495

Fagerhol, M. 65*

Faiman, C. 126

Fatty acid catabolism 211

Fatty acids 487

Faxelius, G. 61*

Fetal age 659

Fetal development 675

Fetal disease 720

Fetuin 894

Fetus 538, 584, 593, 659, 720, 765, 773, 813, $822,887,894$

Fibroblasts 514, 687

Field, M. 65*

Finberg, L. 552

Fine, B. P. 859

Fisher, D. A. 894

Fitzgerald, J. F. 713

Flad, H. D. 65*

Fliedner, T. M. 65*

Fluoride 743

5-Fluorodeoxyuridine 758

Flynn, M. A. 239

Folger, G. M., Jr. 15l

Follicle-stimulating hormone 18, 162, 481

Fondu, P. 57*

Forbes, G. B. 32, 63*, 743

Foulds, J. W. 52*

Fraser, I. D. 52*

Freeman, J. M. 252

Fresco, R. 172

Freska, F. 53*

Friis-Hansen, B. 52*

Gaburro, D. 65*, 880

Gaull, G. 538

Gaull, G. E. 773

Gemes, D. L. 197

Genetic defect 504, 548, 576, 622

Genetic disease 252

Gentz, J. 59*, 64*

Gerald, P. S. 875

Gercker, G. 487

Germain, D. 57*

Gillam, B. M. 773

Gillespie, D. D. 119

Gitzelmann, R. 55*

Glader, B. E. 900

Globoside 606

Gluck, L. 81

Glucocerebrosidase 720

Gluconeogenesis 765

Glucose 182, 765, 813

Glucose metabolism 211

Glucose-6-phosphate dehydrogenase 705

$\alpha$-Glucosidase 803

Glutaminase 797

Glutathione-cystine transhydrogenase 649

Glutathione peridoxidase 900

Gluten-free diet 521
Glycine 576, 622

Glycogen 73, 803

Glycogenolysis 765

$\mathrm{GM}_{2}$ gangliosidosis 606

Golgi apparatus 563

Gonadotropin(s) 18, 126, 481

Graziani, L. J. 203

Greenberg, R. E. 765

Gross, G. P. 593

Growth 32, 126, 239, 521

Growth and development 664

Growth hormone 74:3

Grow th retardation 38, 664, 743

Grumbach, M. M. 162

Gual, C. 481

Gustafson, A. 61*

Gutberlet, R. 61*

Haas, R. J. 65*

Hadorn, H. B. 54*

Hair 868

Hakui, T. 622

Halvorsen, S. 59*

Hambidge, C. 868

Hambidge, K. M. 868

Hanley, W. B. 43

Harada, S. 622

Harries, J. T. 65*

Hathaway, W. E. 593

Hawley, P. 687

Hayes, A. 18

Heart 813,887

Height 32, 239

Heinrich, J. J. 634

Heird, W. C. 495

Heller-Schöch, G. 55*

Heme oxygenase 197

Henderson, J. F. 504

Herod, L. 701

Herschkowitz, N. 66*

Heterophile antibody 145

Hexosaminidase $\rightarrow 606$

Hexosaminidase B 606

Hexosemonophosphate shunt activity 900

Heymann, M. A. 231, 693

Higgins, J. V. 626

Higurashi, M. 514

Hirsch, G. H. 600

Ho, M.-W. 752

Höffken, B. 43

Hollmann, G. 60*

Hollowell, J. G. 151

Homeostasis 219

Homoarginine 626

Homocitrulline 626

Homocystinuria 172, 187

Homograft rejection 779

Homoserine 172

Hoogenraad, N. J. 682

Hook, J. B. 600

Hotes, C. $50^{*}$ 
Hrbek, A. $61^{*}$

Hübner, H. 10

Hull, D. 576

Human growth hormone 664

Hurler's syndrome 563

Hurlet, A. 57*

Hursch, C. J. 529, 643

Hürter, P. 54*

Hyaline membrane disease 81

Hydrogen ion 584

Hydrogen peroxide 900

25-Hydroxycholecalciferol 552

$\beta$-Hydroxyacyl-CoA dehydrogenase 211

5-Hydroxytryptamine 730

Hyman, A. I. 584

Hyperammonemia 252, 622, 875

Hyperglycinemia 622

Hyperglycinemia, ketotic 576

Hypocalcemia 43

Hypogeusia 868

Hypopituitarism, 634, 664

Hypothalamus 162

Hypoxanthine-guanine phosphoribosyltransferase 504

Hypoxemia 203

I-cell disease 752

Imipramine 730

Immunity 779

Immunology 145

Infants $81,219,853$

Ingram, T. T. S. 50 *

Intestine 713,832

Isoenzymes 705

Isomaltase 832

Jacob, J. 752

Jacobs, M. 868

James, L. S. 584

Jeske, J. 10

Johanson, A. 18

Jones, T. C. 548

Jonxis, J. H. P. 58*

Jos, J. 832

Josso, F. 57*

Jouja, V. 51*

Käckell, M. Y. 52*

Kaiser, D. 64*

Kallio, A.-K. 54*

Kang, E. S. 875

Kański, M. 10

Kaplan, S. L. 162

Karacan, I. 529, 643

Karagelli, A. 51*

Karbowski, K. 50*

Karlberg, P. $61^{*}$

Karyotype 514

Kastin, A. J. 481

Katz, M. 52*

Kędziora, J. 10

Keenan, R. E. 111
Kelley, W. N. 504

Kellum, M. 59*, 64*

Khazin, A. F. 81

Kidney 100, 600, 789, 822, 859

Kim, J. K. 600

Kjellmer, I. 61*

Kleihauer, E. 59*

Klein, R. M. 246

Klujber, L. 55*

Knorr, D. $60^{*}$

Kohlberg, I. J. 63*

Kohlschütter, A. 66*

Kohne, E. 59*

Kohrman, A. F. 626

Koprowski, H. 52*

Korenman, S. G. 481

Kosse, W. 53*

Krauss, A. N. 158

Krebs-Henseleit cycle 548, 626

Kretchmer, N. 682

Krivit, W. 56*

Kulin, H. E. 162

Kulovich, M. V. 81

Kuniya, T. 622

Lacourt, G. 63*

Lactate 584

Lactoferrin 69

Lam, R. W. 894

Landman, J. 758

Langenstrassen, H. 50*

Lanman, J. T. 701

Lanning, E. W. 246

Lawrence, R. 63*

Lead 743

Lean body mass 32

Lebowitz, E. A. 887

Lecithin 81, 701

Leisti, S. 62*

Leroy, J. G. 752

Lesch-Nyhan syndrome 504

Leukocyte 649,797

Leung, C. C. K 822

Levine, R. L. 682

Levy, H. L. 548

Lewis substances 853

Leyko, W. 10

Lichte, K. H. 55*

Lindemann, R. 59*

Lipase 880

Lipid(s) 73, 119, 736, 752

Lipolysis 73

Littell, R. C. 529

Liver 26, 100, 197, 548, 622, 675, 773, 797, 875

Lloyd, J. K. $65^{*}$

Lodinová, R. $5 l^{*}$

López, V. 779

Lott, I. T. 730

Low birth weight infants 203

Low birth weight, premature 158
Lucarelli, P. 53*

Lung 81, 675

Luteinizing hormone $18,162,481$

Lymphocyte(s) 514, 779, 803

Lysine 626

Lysosome(s) 563, 649, 752, 803

MacBrinn, M. C. 752

Madigan, P. M. 548

Magnesium 43

Mai, K. 51*

Makowski, E. L. 182

Malnutrition 38, 521

Mamelle, J. C. $63^{*}$

Mann, T. P. 66*

Marasmus 779

Marchi, A. 52*

Marchi, M. A. 52*

Marini, A. $60^{*}$

Marzetti, G. 58*

Massimo, L. 62*

Matsaniotis, N. 59*

Maturation 32, 634

Maurer, H. M. 136

Mautone, A. 58*

McCrea, M. 606

McCulloch, J. R. 720

McMurphy, D. M. 231

Méhes, K. 55*

Melmon, K. L. 231, 693

Menarche 18

Mental retardation $119,504,606,626$

Meschia, G. 182

Metabolic disease 252

Metabolism 813

Metanephrine 151

Methionine 538

Methylmalonic aciduria 875

Methylmalonyl-CoA 875

Methylmalonyl-CoA racemase 875

$N$-Methylnicotinamide 600

Meulen, V. ter $52 *$

Milk 69

Miller, M. C., III 481

Minkowski, A. 57*

Mitochondria 803

Moauro, L. 832

Mohyuddin, F. 54*

Monkus, E. 73, 211

Monkus, E. St. J. 705

Monoamine oxidase 730

Moore, E. S. 859

Mori, P. G. 62*

Morishima, H. O. 584

Morselli, P. L. 53*

Moses, S. W. 55*

Mucopolysaccharide 563

Muller, D. P. R. 65*

Munthe, E. 65*

Murdock, A. I. 60*

Murphy, D. L. 730 
Muscle 219, 813

Neerhout, R. C. 736

Nelson, T. 659

Nelson, W. E. 848

Neonate(s) 43, 211, 487, 593, 600, 693, 705, 713,900

Neth, R. 55*

Neuraminidase 822

Neutron activation 219

Newborn(s) 73, 584

Nicholson, J. F. 252

Nicolopoulos, D. 59*

Nicotinamide-adenine dinucleotide phosphate 705

Niemann-Pick disease 720

Nitrogen metabolism 252

Njai, D. M. 880

Nordio, S. 52*

Norepinephrine 73, 151

Normetanephrine 151

Novak, M. 73, 211

Novick, J. S. 887

Nucleic acid synthesis 682

$5^{\prime}$-Nucleosidase 38

Nucleotides 10

Nutrition 868

Nützenadel, W. 55*

Nyhan, W. L. 1, 576, 705

Oberhänsli-Weiss, I. 693

O'Brien, J. S. 606, 752

Oetliker, O. 54*

Okada, S. 606

Olegård, R. 61*

Olsson, T. 61 *

Omura, K. 622

Orzalesi, M. 53*, 58*

Oseid, S. 59*

Osmolality 495

Ovulation 18

Oxytocin 701

Pajno-Ferrara, F. 65*, 880

Palladini, G. 63*

Palmarino, R. 53*

Palmitate-1 $1{ }^{14} \mathrm{C} 675$

Pantelakis, S. N. 52*

Pantlitschko, M. 53*

Papadatos, C. 51*, 52*

Papaevangelou, G. 51*

Parathyroid hormone 552

Parenteral alimentation 789

Parkinson, D. K. 43

Parturition 701

Pascal, T. A. 773

Paunier, L. 63*

Pearson, H. A. 145

Penile erections 529

Pennock, C. A. 52*

Perez-Pasten, E. 481
Perheentupa, J. 62*, 797

Persson, B. 59* 64*

Pfeiffer, R. A. 62*

Phagocytosis 563

Phenobarbital 100

Phosphatidyl choline 736

Phosphofructokinase 211

Phospholipids 81, 487, 675, 701

Phosphorus 43

Photodecomposition 246

Phototherapy 246

Pigram, P. 53*

Pineda, G. 203

Pious, D. A. 687

Pituitary 126, 162

Placenta 675

Platelets 136

Podestá, E. J. 634

Pojerova, A. $63^{*}$

Poláček, K. 64*

Potassium 239

Potassium transport 616

Prader, A. 521

Premature 880

Prematurity 231, 693

Price, T. 495

Primates 548

Principi, N. 53*

Propionate 576, 875

Propionyl-CoA carboxylase 576

Proteinuria 822

Puberty 126, 162, 481, 529, 634, 643

Pyridoxine 187

Pyrimidine biosynthesis 682

Rabo, E. 53*

Radde, I. C. 43

Radiosensitivity, chromosomal 514

Rager, K. 54*

Räihä, N. C. R. 538

Rasmussen, K. 576

Raye, J. 61*

Reina, J. C. 643

Reiser, S. 713

Releasing factor 481

REM sleep 529

Renal transport 600

Respiration, cellular 687

Respiratory distress syndrome 81

Rey, F. 832

Rey, J. 832

Rhesus factor 487

Rickets 552

Riegel, K. P. $60^{*}$

Rivarola, M. A. 634

Robbins, J. B. 69

Rodier, P. 758

Rose bengal 100

Rosen, J. F. 552

Rosenberg, L. E. 187

Rossi, E. 64*
Rossner, A. K. 55*

Roux, J. F. 675

Rubino, A. 65*

Rudolph, A. M. 231, 693, 887

Russell, A. $51^{*}$

Sagerson, R. N. 659

Saliva 853

Sandhoff's disease 606

Sandstead, H. H. 119

Sarcia, S. R. 584

Satrasook, S. S. 859

Schadwinkel, R. $60^{*}$

Schalch, D. 63*

Schalch, D. S. 48I

Schally, A. V. 481

Schärer, K. 55*

Schaub, J. 53*

Scheffner, D. 50*

Schettini, F. 58*

Schmidt, H. D. 60*

Schneider, E. L. 720

Schneider, H. 50*

Schneider, J. M. 182

Schöber, J. 60*

Schröter, W. 487

Schulman, J. D. 649

Schulte, F. J. 50*

Schwartz, A. D. 145

Scott, C. R. 659

Scott-Emuakpor, A. 626

Scriver, C. R. 54*

Seashore, M. R. 187

Sereni, F. 53*

Serine 576

Serotonin 730

Sexual development 1 Il

Sexual infantilism 634

Sexual maturation 126

Sharp, H. 56*

Sherman, D. 563

Shih, V. E. 548

Shimada, M. 758

Shmerling, D. H. 521

Shwachman, H. 803

Sialic acid 69

Sickle cell anemia 145

Siltanen, I. 54*

Simcha, A. $5 l^{*}$

Simell, O. 797

Sippell, W. G. 803

Sizonenko, P. C. 63*

Skardoutsou, A. 51*

Skin 219

Slaunwhite, W. R., Jr. 111

Sleep 529, 643

Sleep, stages of 643

Smith, N. J. 779

Snodgrass, P. J. 875

Snyder, P. 56*

Soderlund, S. 56* 
Sodium reabsorption 859

Sodium transport 616

Solc, J. 60*

Sфvik, O. 59*

Spennati, G. F. 53*

Sphingolipid 720

Sphingomyelin 736

Sphingomyelinase 720

Spleen 145, 197

Spycher, M. A. 55*

Stahlman, M. 61*

Starch 832

Stave, U. 211

Sterols 675

Stolzis, L. 50*

Strontium 743

Stubbe, P. 63*

Sturgeon, P. 853

Sturman, J. A. 538

Sucrase 832

Surfactant 81

Suwatanawiroy, A. 60*

Swanstrom, S. 61*

Sympathetic innervation 887

Sympathetic nervous system 693

Tada, K. 622

Takada, G. 622

Taylor, M. R. H. 62*

Tay-Sachs disease 606

Teratology 822

Testicle 634

Testosterone 111, 126, 162, 634

Tetracycline 687

Thaler, M. M. 100, 197

THAM 495

Thau, R. 701

Thodenius, K. 64*

Thouverez, J. P. 57*
Thrombocytes 730

Thyroid hormone 664

Thyroxine 894

Thyroxine-binding protein 894

Tietze, F. 649

Tiling, T. 487

Tognoni, G. 53*

Torroni, A. 58*

Trace elements 868

Transport 26

Transport, amino acid 713

Triglycerides 675

Triiodothyronine 894

Trisomy G 10

Tryphonopoulou, Y. 51*

Trypsin 880

Tsomides, K. 59*

Tsoulos, N. G. 182

Underwood, B. A. 26

Urban, A. E. 60 *

Urea cycle 797

Urea metabolism 252

Urea synthesis 548

Uric acid 504

Uridine kinase 682

Valaes, T. 52*

Vanillylmandelic acid 151

Varela-Cives, $60^{*}$

Vassella, F. 50*

Ventilation perfusion ratio 158

Vergel, Z. M. 859

Viala, J. J. 57*

Victorin, L. 61*

Virág, I. 57*

Visakorpi, J. K. 797

Visual evoked response 203

Vitamin A 26
Vitamin D 552

Vitamin E 26

Wada, Y. 622

Wagner, V. 51*

Walker, C. H. M. 61*

Wallace, S. J. 50*

Water equilibrium 219

Weichsel, M. E., Jr. 682

Weight 239

Weippel, G. 53*

Weisser, K. 60*

Weitzman, E. D. 203

Wharton, B. A. 52*

Wheeler, W. E. 840

Wick, H. 56*

Wiesmann, U. 66*

Williams, R. L. 529, 643

Winkler, K. 55*

Winter, J. S. D. 126

Winters, R. W. 495

Wolf, H. 63*, 211

Wolfson, D. R. 69

Wong, P. W. K. 172

Woodruff, C. 239

Wyler, F. 60*

Xanthou, M. 59*

Yoshida, T. 622

Yoshioka, T. 675

Young, M. K. 813

Zahnd, G. 63*

Zetterström, R. 64*

Zielke, K. 752

Zinc 119, 868

Zoppi, G. 65*, 880

Zwinger, A. 64* 